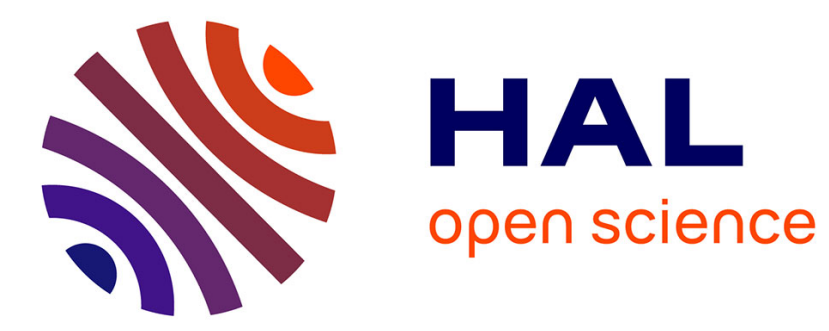

\title{
Modifications de la croissance et de l'intensité respiratoire de racines de blé infectées par Heterodera avenae Woll
}

Jacques Davy de Virville, Michèle Chauveau, Françoise Person-Dedryver

\section{To cite this version:}

Jacques Davy de Virville, Michèle Chauveau, Françoise Person-Dedryver. Modifications de la croissance et de l'intensité respiratoire de racines de blé infectées par Heterodera avenae Woll. Agronomie, 1984, 4 (9), pp.813-818. hal-00884701

\section{HAL Id: hal-00884701 \\ https://hal.science/hal-00884701}

Submitted on 1 Jan 1984

HAL is a multi-disciplinary open access archive for the deposit and dissemination of scientific research documents, whether they are published or not. The documents may come from teaching and research institutions in France or abroad, or from public or private research centers.
L'archive ouverte pluridisciplinaire HAL, est destinée au dépôt et à la diffusion de documents scientifiques de niveau recherche, publiés ou non, émanant des établissements d'enseignement et de recherche français ou étrangers, des laboratoires publics ou privés. 


\section{Modifications de la croissance et de l'intensité respiratoire de racines de blé infectées par Hete- rodera avenae Woll.}

Jacques DAVY de VIRVILLE, Michèle CHAUVEAU \& Françoise PERSON-DEDRYVER (*)

C.N.R.S. (E.R.A. 1043), Laboratoire de Biologie végétale IV. Université Pierre et Marie Curie, 12, rue Cuvier, F 75005 Paris

(*) I.N.R.A., Station de Zoologie, Centre de Recherches de Rennes, B.P. 29, F 35650 Le Rheu

RÉSUMÉ

La croissance et l'évolution de l'intensité respiratoire des racines de 2 cultivars de blé (Triticum aestivum L.) ont été suivies après 4, 8, 15 et 21 jours d'infection par le nématode Heterodera avenae Woll. Les études ont porté sur un cultivar sensible ( Capitole ») et sur un cultivar résistant («Loros »).

Pour "Capitole ", l'infection entraîne d'abord un ralentissement de l'élongation des racines, puis celui de l'accroissement de matière sèche. La reprise de la croissance devient importante au $15^{\mathrm{e}}$ jour, où se produit une stimulation de l'intensité respiratoire des tissus. Pour « Loros ", l'inhibition de la croissance, particulièrement marquée, s'accompagne au $8^{\mathrm{e}}$ jour d'un ralentissement très important de l'activité respiratoire des racines. Une faible reprise de la croissance intervient tardivement pour ce cultivar ; en même temps a lieu une légère stimulation de l'intensité respiratoire.

Les résultats suggèrent que l'action néfaste du nématode est plus liée à la multiplicité des sites d'infection et au ralentissement de croissance qui en résulte qu'à un effet directement inhibiteur sur le métabolisme des tissus. D'autre part, il semble que la résistance au nématode ne se traduise pas en début d'infection par le déclenchement d'un mécanisme de type hypersensibilité, la stimulation observée de l'intensité respiratoire étant plutôt liée à la reprise de la croissance racinaire et de l'activité méristématique. Par contre, le ralentissement marqué de l'activité métabolique des tissus infectés, tel qu'il existe dans le cas de "Loros ", pourrait jouer un rôle important dans l'expression de la résistance des tissus.

Mots clés additionnels : Triticinées, respiration, résistance. Woll.

Changes in growth and respiratory rates in the roots of 2 cultivars of wheat (Triticum aestivum L.) were measured $4,8,15$ and 21 days after infection by the nematode Heterodera avenae Woll. One cultivar ("Capitole") was susceptible to the pathogen and the other ("Loros") resistant.

For "Capitole", infection reduced root elongation first and then dry weight increase. Growth resumed at the 15 th day, concomitantly with a stimulation of respiratory activity. For "Loros", growth inhibition was more severe, with a major decrease in root respiration at day 8. A slight resumption of growth took place later than with "Capitole", as well as a slight increase of respiration.

These results indicate that the damaging effects of the pathogen are mostly linked to the number of infection sites and to a concomitant reduction in growth rate rather than to a direct inhibitory effect on metabolism. However, it appears that the main factor for resistance to the nematode does not correspond, in the early period of infection, to the setting up of a mechanism of hypersensitivity : the increase in respiratory activity appeared to be mainly linked to a resumption of root growth and meristem activity. For " Loros ", a slowing down of metabolic activity in infected tissues might play a major role in the expression of tissue resistance.

Additional key words : Triticinae, respiration, resistance.

\section{INTRODUCTION}

Heterodera avenae Woll. est le nématode causant le plus de dégâts aux cultures céréalières de par sa répartition géographique et sa gamme d'hôtes (RITTER,
1982). Il existe peu de données globales permettant d'évaluer l'importance des dommages, car ceux-ci sont souvent le fait d'une association avec d'autres nématodes ou même avec des affections cryptogamiques. Ils peuvent varier également avec la nature et les con- 
ditions de développement de la plante (CAUBEL et al., 1980). On estime cependant que les pertes de rendement peuvent être de l'ordre de 50 p. 100 dans les cas les plus graves et au moins de l'ordre de 5 à 10 p. 100 dans les zones céréalières en France (RITTER, 1972). L'extension des cultures céréalières, l'utilisation de cultivars performants, la tendance à la monoculture de céréales à paille favorisent le pullulement de ce parasite (CAUBEL et al., 1980 ; RITTER, 1982).

L'étude des principales phases de développement de ce nématode (SOSA MOSS, 1966), la caractérisation en France de 4 pathotypes définis par leur gamme d'hôtes (RIVOAL, 1977 ; PERSON-DEDRYVER \& PANNETIER, 1982) ou par leurs conditions de développement (RIVOAL, 1978, 1979) et la mise au point de systèmes d'infection artificiels en champ ou en conditions de laboratoire (RIVOAL et al., 1978) permettent maintenant de mieux étudier ce parasite. Aussi, comme il n'existe actuellement aucune donnée d'ordre physiologique, a-t-il paru intéressant de suivre l'évolution de différents paramètres permettant d'évaluer le développement et l'activité métabolique des tissus infectés afin de déterminer l'existence de réactions tissulaires en relation avec le caractère de sensibilité ou de résistance de la plante vis-à-vis du nématode.

\section{MATÉRIEL ET MÉTHODES}

\section{A. Matériel biologique}

Pour suivre cette évolution, 2 cultivars de blé (Triticum aestivum $\mathbb{L}$.) ont été choisis :

" Capitole », cultivar sensible de grande culture, qui permet la multiplication facile du nématode,

« Loros », cultivar résistant sans intérêt agronomique, issu du croisement « Koga II » $x$ « CI 3779 », qui n'autorise qu'une faible multiplication du parasite et peut servir de géniteur de résistance.

Outre ce comportement très différent, ces 2 cultivars ont été également retenus en raison de l'évolution bien connue des symptômes induits par la présence du nématode (PERSON \& DoussinaulT, 1978).

Les infections sont réalisées au moyen de larves du $2^{e}$ stade d' $H$. avenae obtenues à partir de kystes provenant de sol infecté ; ceux-ci sont conservés dans l'eau durant 2 mois à la température de $20{ }^{\circ} \mathrm{C}$ puis placés à $5{ }^{\circ} \mathrm{C}$ afin de lever leur diapause (RIvOAL, 1978).

Les infections sont réalisées à la station de l'I.N.R.A. de Rennes; les études physiologiques sont effectuées au Laboratoire de Biologie Végétale IV, à Paris. Pour éviter une perte d'agressivité au cours du transport, le pathotype d' $H$. avenae utilisé a été choisi en raison de sa moindre sensibilité à la température (PERSON \& DOUSSINAUlT, 1978). Il s'agit du pathotype FRI selon la classification de RIVOAL (1977). Au total, ce travail a nécessité l'emploi d'environ 12000 larves.

\section{B. Techniques de culture et d'inoculation}

Les infections sont réalisées selon la technique de PERSON \& DOUSSINAULT (1978), largement utilisée en sélection pour tester le comportement des plantes visà-vis du nématode. Elle présente l'avantage de fournir des résultats similaires à ceux obtenus en conditions de plein champ, en éliminant les risques d'intervention d'autres parasites telluriques. Les caryopses sont mis à germer et, au stade 3 racines développées, ils sont placés en boîtes de Petri. Seize larves sont alors déposées par racine, soit 48 larves par caryopse. Les boîtes sont conservées pendant $48 \mathrm{~h}$ à $20^{\circ} \mathrm{C}$, température optimale pour l'obtention d'une bonne pénétration des larves dans la racine (DAVIES \& FISCHER, 1976). Elles sont ensuite placées dans des enceintes climatisées maintenues à $18 \pm 1{ }^{\circ} \mathrm{C}$, sous un éclairage de 2000 lux environ pendant $15 \mathrm{~h}$ par jour.

\section{Mesure de l'intensité respiratoire}

Les mesures d'intensité respiratoire ont été effectuées $4,8,15$ et $21 \mathrm{j}$ après l'infection, c'est-à-dire aux temps $7,11,18$ et $24 \mathrm{j}$ à compter du début de la germination des caryopses. Ces différents temps correspondent aux principales phases du développement du nématode, telles qu'elles ont été définies par SosA Moss (1966). Il aurait été intéressant de poursuivre les mesures pendant 1 mois, période qui correspond au développement complet des femelles fécondées. En pratique, une durée de $21 \mathrm{j}$ représente le temps maximal pendant lequel les racines peuvent être prélevées. En effet, à ce stade, le milieu gélosé commence à s'infecter ou même à se dessécher, ce qui rend très difficile la séparation de la racine du milieu gélosé.

De 25 à 35 caryopses ont été étudiés séparément par condition et par stade de croissance. A chaque stade, les racines sont retirées de la gélose puis lavées à l'eau courante. Au cours de la croissance, 2 autres racines séminales peuvent se développer, mais elles ne sont pas infectées. Ces 2 racines sont éliminées systématiquement. L'intensité respiratoire des 3 racines principales, excisées juste avant les mesures, est alors immédiatement mesurée en milieu liquide agité $(2 \mathrm{ml})$ à l'aide d'une électrode de Clark à oxygène en présence de tampon phosphate $20 \mathrm{mM}(\mathrm{pH} 7,2)$, à $25^{\circ} \mathrm{C}$. Après mesure de l'intensité respiratoire, la longueur des racines et la masse de matière fraîche sont déterminées. La masse de matière sèche est ensuite obtenue après dessiccation du matériel à $105^{\circ} \mathrm{C}$ pendant $7 \mathrm{j}$.

\section{RÉSULTATS}

Pour mesurer les modifications du métabolisme induites par le parasite, plusieurs paramètres ont été retenus : d'une part, la longueur totale des racines primaires, qui permet d'évaluer les modifications de l'élongation (les racines secondaires restant très peu développées même après $24 \mathrm{j}$ de culture) ; d'autre part, les masses de matières fraîche (MF) et de matière sèche (MS), qui rendent compte de l'hydratation des tissus et des modifications de la croissance pondérale, c'est-à-dire essentiellement des synthèses de parois ; enfin, l'activité respiratoire, mesurée et exprimée par système racinaire (3 racines par caryopse) et par $\mathrm{g}$ de MS. Dans ce qui suit, les temps sont comptés soit à partir de la germination des caryopses $(t)$, soit à partir de l'infection ( $t-3 \mathrm{j})$. 


\section{A. Comportement du matériel témoin}

\section{Croissance des racines}

Les évolutions de la longueur et de la masse de matière sèche des racines des 2 cultivars "Capitole " et «Loros" sont représentées dans les figures $1 \mathrm{~A}$ et $1 \mathrm{~B}$.

Les coefficients de régression $(r)$ proches de 1 montrent que, pendant la période étudiée, les évolutions de ces 2 paramètres peuvent être considérées comme linéaires. Les vitesses d'élongation et de croissance pondérale représentées par les pentes de ces droites peuvent être alors calculées. Elles sont respectivement de 1,31 et de $0,46 \mathrm{mg} \mathrm{MS} / \mathrm{j}$ pour « Capitole ». Elles sont par contre de 0,76 et de $0,24 \mathrm{mg} \mathrm{MS} / \mathrm{j}$ dans le cas de «Loros ", c'est-à-dire 2 fois plus faibles que pour « Capitole».

Enfin, l'hydratation des racines a été estimée par le rapport MF/MS (fig. $1 \mathrm{C}$ ). Ces rapports sont constants, quel que soit le stade de croissance, et identiques pour les 2 cultivars. Leur valeur moyenne est de l'ordre de 11 à 12 , ce qui représente 10 à $11 \mathrm{~g}$ d'eau pour $1 \mathrm{~g}$ de MS.
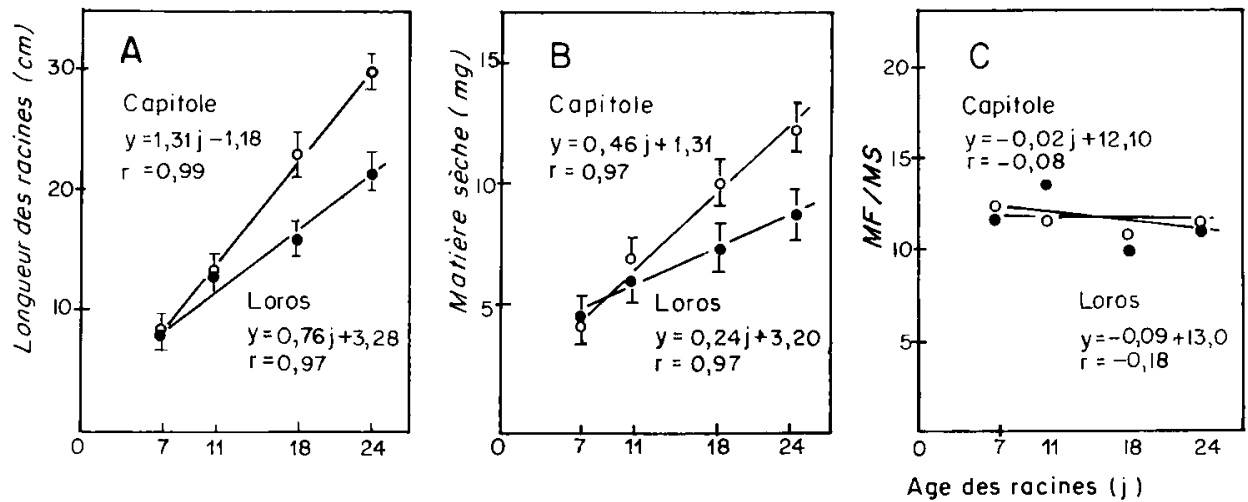

Figure 1

Evolution au cours du temps de la longueur ( $\mathrm{cm} /$ racine), de la masse de matière sèche ( $\mathrm{mg} / 3$ racines) et de l'hydratation (MF/MS) des racines de 2 cultivars de blé sensible ("Capitole ») ou résistant ("Loros») à Heterodera avenae Woll.

Les barres correspondent à l'écart-type à la moyenne obtenue pour chaque valeur.

Changes in length ( $\mathrm{cm} /$ root), dry matter ( $\mathrm{mg} / 3$ roots) and hydration (fresh weight/dry weight) of roots of two cultivars of wheat susceptible ("Capitole") or resistant ("Loros") to Heterodera avenae Woll.

Bars are standard deviations of the means.

\section{Evolution de l'intensité respiratoire}

Les résultats concernant l'évolution de l'intensité respiratoire sont présentés dans le tableau 1. Pour "Capitole ", l'intensité respiratoire du système racinaire est la même aux $7^{\mathrm{e}}, 18^{\mathrm{e}}$ et $24^{\mathrm{e}} \mathrm{j}$. Par contre, on observe de manière constante un maximum dans la respiration des racines au $11^{\mathrm{e}} \mathrm{j}$; l'intensité respiratoire est à ce stade environ 1,5 fois plus élevée qu'aux autres stades de croissance. Cependant, il y a diminution de l'intensité respiratoire rapportée à la MS de l'ordre d'un facteur 4 en relation avec l'augmentation de la quantité de MS synthétisée au cours de la croissance des racines.

L'intensité respiratoire des racines de «Loros » est assez semblable à celle de "Capitole ". La respiration des tissus présente aussi un optimum au $11^{\mathrm{e}} \mathrm{j}$. Il y a également diminution de l'intensité respiratoire par $\mathrm{g}$ de MS, mais de manière moins importante que dans le cas de "Capitole ». L'intensité respiratoire est donc plus importante dans ce cas, surtout à partir du $18^{\mathrm{e}} \mathrm{j}$.

On voit donc que, malgré une capacité de croissance 2 fois plus faible de "Loros " par rapport à "Capitole ", les activités respiratoires des 2 cultivars sont très voisines.
TABLEAU 1

Evolution au cours du temps de l'intensité respiratoire des racines de 2 cultivars de blé sensible ("Capitole ") ou résistant ("Loros ") à Heterodera avenae Woll.

Time course changes in the respiratory activity of roots of two cultivars of wheat susceptible (" Capitole ») or resistant ("Loros ») to Heterodera avenae Woll.

\begin{tabular}{ccc}
\hline \multirow{2}{*}{$\begin{array}{c}\text { Age des } \\
\text { racines }\end{array}$} & \multicolumn{2}{c}{ Intensité respiratoire } \\
\cline { 2 - 3 } & $\mathrm{QO}_{2}{ }^{\left({ }^{a}\right)}$ & $\mathrm{QO}_{2} \mathrm{MS}$ ( $\left.^{\mathrm{b}}\right)$ \\
\hline Capitole & & \\
\hline $7 \mathrm{j}$ & 15 & 3850 \\
$11 \mathrm{j}$ & 22 & 2580 \\
$18 \mathrm{j}$ & 15 & 1200 \\
$24 \mathrm{j}$ & 13 & 1070 \\
Loros & & \\
\hline $7 \mathrm{j}$ & 17 & 3700 \\
$11 \mathrm{j}$ & 22 & 3500 \\
$18 \mathrm{j}$ & 11 & 1500 \\
$24 \mathrm{j}$ & 13 & 1500 \\
\hline
\end{tabular}

(a) $\mu \mathrm{l} \mathrm{O}_{2} / \mathrm{h} / 3$ racines. (b) $\mu \mathrm{l} \mathrm{O}_{2} / \mathrm{h} / \mathrm{g}$ de matière sèche. L'écarttype à la moyenne des valeurs rapportées dans ce tableau est de l'ordre de 10 p. 100. 


\section{B. Comportement du matériel infecté}

Les résultats sont rapportés dans le tableau 2. Pour mieux évaluer l'effet de l'infection, les rapports des valeurs observées chez le matériel infecté à celles mesurées chez le matériel sain ont été également indiqués (chiffres entre parenthèses). Une valeur supérieure à 1 montrera un effet stimulateur de l'infection et une valeur inférieure à 1 un effet inhibiteur.

\section{Croissance des racines}

Pour « Capitole », après $4 \mathrm{j}$ d'infection, le principal symptôme correspond à un ralentissement important de l'élongation des racines infectées. Après $8 \mathrm{j}$, quelques racines commencent à se développer à partir de la zone d'infection; elles sont nombreuses après le $15^{\mathrm{e}} \mathrm{j}$, mais leur élongation ne devient importante qu'après le $21^{\mathrm{e}} \mathrm{j}$. Après le $4^{\mathrm{e}} \mathrm{j}$, l'hétérogénéité morphologique des racines rend donc impossible la mesure de l'élongation par l'estimation de la longueur totale des racines.

L'évolution des différents critères de croissance est présentée dans le tableau 2 , où seule a donc été rapportée la longueur des racines après $4 \mathrm{j}$ d'infection. On voit effectivement que l'inhibition est importante à ce stade d'infection ( 30 p. 100). En ce qui concerne la masse de MS, il y a encore, comme pour le matériel sain, augmentation de la MS des racines au cours des $21 \mathrm{j}$ d'infection. On voit cependant que l'infection exerce un effet inhibiteur à partir du $8^{\mathrm{e}} \mathrm{j}$ et surtout au $15^{\mathrm{e}} \mathrm{j}$, où la quantité de MS est environ $30 \mathrm{p} .100$ inférieure à celle du témoin. Cet effet inhibiteur est ensuite partiellement levé après le $21^{\mathrm{e}} \mathrm{j}$. En ce qui concerne l'hydratation des racines infectées, on voit qu'elle est alors sensiblement augmentée, principalement au $8^{\mathrm{e}}$ et au $15^{\mathrm{e}} \mathrm{j}$ (de 35 à $40 \mathrm{p} .100$ ).

Pour "Loros ", on assiste après $4 \mathrm{j}$ d'infection au ralentissement de l'élongation des racines, laquelle est ensuite complètement bloquée entre le $4^{\mathrm{e}}$ et le $8^{\mathrm{e}} \mathrm{j}$. Contrairement à "Capitole», aucune nouvelle formation de racines ne peut être observée à ce stade d'infection. Après $15 \mathrm{j}$, seules quelques racines commencent à se développer à partir du point d'infection. A $21 \mathrm{j}$, le nombre de ces racines reste faible (elles sont en particulier beaucoup moins nombreuses que dans le cas de " Capitole »), par contre leur élongation peut devenir importante. Les mesures rapportées dans le tableau 2 soulignent effectivement l'arrêt de l'allongement racinaire à partir du $4^{\mathrm{e}} \mathrm{j}$ et ensuite sa reprise du $8^{\mathrm{e}}$ au $21^{\mathrm{e}} \mathrm{j}$. L'inhibition de l'élongation par rapport au témoin est importante jusqu'au $8^{\mathrm{e}} \mathrm{j}(60 \mathrm{p}$. 100) ; elle est encore de 50 p. 100 au $21^{\mathrm{e}} \mathrm{j}$. La reprise de l'élongation observée reste donc globalement faible. Malgré cet arrêt de l'élongation, il y a encore augmentation du poids de MS, mais de manière moins marquée que pour le témoin ; il y a en effet inhibition au $15^{\mathrm{e}}(21 \mathrm{p} .100)$ et surtout au $21^{\mathrm{e}} \mathrm{j}(27 \mathrm{p} .100)$. Enfin, en ce qui concerne l'hydratation des tissus, elle n'est pas touchée et demeure à peu près constante quelle que soit la durée de l'infection. Elle est alors sensiblement plus faible que dans le cas de "Capitole».

\section{Intensité respiratoire}

Les mesures sont rapportées dans le tableau 2. Pour "Capitole ", on observe, comme pour le matériel sain, un optimum de la respiration au $8^{\mathrm{e}} \mathrm{j}$; cependant, dans l'ensemble, l'intensité respiratoire demeure à peu près constante. Dans tous les cas, elle est cependant légèrement inférieure à celle du témoin (environ 10 p. 100). En ce qui concerne l'intensité respiratoire relative au g de MS, elle va en diminuant du $4^{e}$ au $21^{\mathrm{e}} \mathrm{j}$. Cependant, par rapport au témoin, on voit qu'après une inhibition d'environ $20 \mathrm{p} .100$ au $4 \mathrm{e}$ j, l'activité respiratoire devient même supérieure d'environ $30 \mathrm{p} .100$ au $15^{\mathrm{e}} \mathrm{j}$. Cet effet stimulateur exercé par l'infection n'est visible que transitoirement, car au

\section{TABLEAU 2}

Evolution au cours du temps de la longueur, de la masse de MS, de l'hydratation et de l'intensité respiratoire des racines de 2 cultivars de blé sensible (« Capitole ») ou résistant («Loros ») infecté par Heterodera avenae Woll.

Time course changes of length, dry matter, hydration and respiratory rates of roots of two cultivars of wheat, susceptible (« Capitole ») or resistant («Loros »), infected with Heterodera avenae Woll.

\begin{tabular}{|c|c|c|c|c|c|}
\hline \multirow{2}{*}{ Age des racines } & \multirow{2}{*}{ Longueur (a) } & \multirow{2}{*}{ Matière sèche $\left({ }^{b}\right)$} & \multirow{2}{*}{$\mathrm{MF} / \mathrm{MS}$} & \multicolumn{2}{|c|}{ Intensité respiratoire } \\
\hline & & & & $\mathrm{QO}_{2}(\mathrm{C})$ & $\mathrm{QO}_{2} \mathrm{MS}(\mathrm{d})$ \\
\hline \multicolumn{6}{|l|}{ Capitole } \\
\hline $7 j(4 j)$ & $5,8(0,71)$ & $4,2(1,05)$ & $14,5(1,18)$ & $13(0,86)$ & $3350(0,82)$ \\
\hline $11 \mathrm{j}(8 \mathrm{j})$ & - & $6,0(0,85)$ & $16,0(1,36)$ & $20(0,91)$ & $2680(1,03)$ \\
\hline $18 \mathrm{j}(15 \mathrm{j})$ & - & $7,2(0,71)$ & $16,0(1,42)$ & $14(0,90)$ & $1560(1,30)$ \\
\hline $24 \mathrm{j}(21 \mathrm{j})$ & - & $10,3(0,85)$ & $14,8(1,22)$ & $12(0,92)$ & $1140(1,08)$ \\
\hline \multicolumn{6}{|l|}{ Loros } \\
\hline $7 \mathrm{j}(4 \mathrm{j})$ & $5,5(0,69)$ & $3,8(0,83)$ & $12,3(1,07)$ & $16(0,94)$ & $4180(1,13)$ \\
\hline $11 \mathrm{j}(8 \mathrm{j})$ & $5,1(0,39)$ & $5,8(0,94)$ & $11,6(0,84)$ & $12(0,55)$ & $2070(0,59)$ \\
\hline $18 \mathrm{j}(11 \mathrm{j})$ & $7,5(0,47)$ & $5,9(0,79)$ & $10,3(1,07)$ & $9(0,79)$ & $1500(1,00)$ \\
\hline $24 \mathrm{j}(21 \mathrm{j})$ & $11,0(0,50)$ & $6,4(0,73)$ & $12,3(1,09)$ & $11(0,85)$ & $1740(1,16)$ \\
\hline
\end{tabular}

(a) $\mathrm{cm} /$ racine. (b) $\mathrm{mg} / 3$ racines. ( $\left.{ }^{c}\right) \mu \mathrm{l} \mathrm{O}_{2} / \mathrm{h} / 3$ racines. $\left({ }^{\mathrm{d}}\right) \mu \mathrm{l} \mathrm{O}_{2} / \mathrm{h} / \mathrm{g}$ de M.S. Le nombre de jours après infection est indiqué à la suite de l'âge des racines. Les valeurs entre parenthèses sont les rapports des mesures effectuées sur les plantes infectées et sur les plantes saines. L'écart-type à la moyenne relatif à la longueur des racines est de $4 \mathrm{p}$. 100. Celui relatif aux autres paramètres est de l'ordre de $10 \mathrm{p}$. 100 . 
$21^{\mathrm{e}} \mathrm{j}$, l'intensité respiratoire relative au g de MS redevient comparable à celle du témoin.

En ce qui concerne «Loros », on remarque qu'il n'y a pas d'optimum de respiration au $8^{\mathrm{e}} \mathrm{j}$ et que l'intensité respiratoire est alors plus élevée au $4^{e} j$ d'infection qu'aux autres stades. A $8 \mathrm{j}$, il y a donc inhibition de manière très marquée $(45 \mathrm{p}$. 100). Cette inhibition est ensuite levée progressivement du $8^{\mathrm{e}}$ au $21^{\mathrm{e}} \mathrm{j}$, où elle n'est plus que de $15 \mathrm{p}$. 100. Comme pour les autres conditions, il y a diminution au cours du temps de l'intensité respiratoire exprimée par rapport à la matière sèche. On voit alors que par rapport au témoin, la respiration est légèrement stimulée $a u 4^{e} \mathrm{j}$; mais elle est surtout nettement inhibée au $8^{\mathrm{e}} \mathrm{j}$ (40 p. 100). L'inhibition est levée par la suite et on observe même une légère stimulation au $21^{\mathrm{e}} \mathrm{j}$ en raison de la diminution relative de la teneur en MS des tissus infectés.

En conclusion, les résultats suivants peuvent être retenus: dans les racines des plantules de « Capitole », au $4^{\mathrm{e}} \mathrm{j}$ d'infection, la croissance pondérale comme l'intensité respiratoire ne sont pas affectées bien que l'élongation des racines soit alors fortement ralentie. Entre le $8^{\mathrm{e}}$ et le $15^{\mathrm{e}} \mathrm{j}$, l'hydratation des tissus augmente fortement, alors que la croissance pondérale se ralentit sensiblement jusqu'au $15^{\mathrm{e}} \mathrm{j}$, où une stimulation importante de l'intensité respiratoire peut être observée. Cette stimulation correspond au moment où de nombreuses petites racines se développent à partir du point d'infection. Après le $21^{\mathrm{e}} \mathrm{j}$, les valeurs prises par les différents paramètres se rapprochent sensiblement de celles mesurées chez les racines témoins. Le développement important de nouvelles racines vient donc compenser quantitativement le retard de croissance lié à l'infection.

Pour «Loros ", après le $4^{\mathrm{e}}$ comme après le $8^{\mathrm{e}} \mathrm{j}$, l'élongation des racines est fortement ralentie alors que la croissance pondérale n'est pas diminuée. Ces 2 paramètres sont donc affectés comme pour «Capitole ", mais de manière plus marquée et plus tardive (jusqu'au $8^{\mathrm{e}} \mathrm{j}$ ). De plus, dans le cas de "Loros", l'arrêt complet de l'élongation s'accompagne au $8^{\mathrm{e}} \mathrm{j}$ d'un ralentissement très important de l'activité métabolique des tissus. Après le $8^{\mathrm{e}} \mathrm{j}$, la croissance pondérale se ralentit et est essentiellement inhibée après le $21^{\mathrm{e}} \mathrm{j}$ (30 p. 100). Ce ralentissement correspond au retard de développement des racines infectées. La croissance racinaire reprend à partir du $15^{\mathrm{e}} \mathrm{j}$, moins toutefois que pour «Capitole ». En même temps se produit une levée de l'inhibition de l'intensité respiratoire et même une légère stimulation au $21^{\mathrm{e}} \mathrm{j}$.

\section{DISCUSSION}

La comparaison des résultats montre qu'il existe une certaine analogie entre les modifications métaboliques et morphologiques observées en réponse au développement du nématode dans les tissus des racines du cultivar sensible «Capitole » comme dans ceux du cultivar résistant « Loros ».

En fait, la principale différence de comportement entre les 2 cultivars correspond à l'existence, pour le cultivar sensible, d'une stimulation de la respiration nettement plus marquée (au $15^{\mathrm{e}} \mathrm{j}$ ), et, pour le cultivar résistant, d'un ralentissement plus important de l'élongation et surtout de l'activité métabolique des tissus au $8^{\mathrm{e}} \mathrm{j}$.

On voit, d'autre part, qu'il y a un décalage important dans l'apparition des symptômes, puisque le ralentissement de l'élongation, le développement de nouvelles racines et la stimulation de l'intensité respiratoire surviennent plus tardivement dans le cas de " Loros ».

Deux points principaux sont donc à souligner : la signification de la stimulation de la respiration observée $\mathrm{au} 15^{\mathrm{e}} \mathrm{j}$ et celle du ralentissement de l'activité métabolique des tissus infectés.

La stimulation de l'intensité respiratoire d'un tissu en réponse à une infection est un phénomène assez général (BECKMAN, 1964 ; GOODMAN et al., 1967 ; WHEELER, 1975 ; DALY, 1976). Cette stimulation peut correspondre au déclenchement des mécanismes de résistance, mais elle est souvent d'autant plus importante (et précoce) que le degré de résistance de la plante l'est également. Le contraire est observé ici, la stimulation de la respiration étant plus précoce et plus intense dans le cas de «Capitole». Un tel résultat pourrait s'expliquer par un retard à la pénétration du parasite tel qu'il existe dans le cas d'une autre maladie du blé, le piétin-verse (JAHIER, 1978 ; DAVY de VIRVILLE et al., 1982). En fait, un tel phénomène n'est généralement pas considéré comme jouant un rôle dans le cas des infections à nématodes (KAPLAN \& KEEN, 1980). On voit d'ailleurs, dans le cas présent, que les retards de croissance consécutifs à la pénétration du nématode sont tout à fait comparables après le $4^{\mathrm{e}} \mathrm{j}$ d'infection pour les 2 cultivars. De plus, chez «Loros », la réaction vis-à-vis de l'infection, maximum au $8^{\mathrm{e}} \mathrm{j}$, entraîne au contraire une inhibition de l'intensité respiratoire. Enfin, l'examen des racines ne montre l'existence d'une légère nécrose et d'un brunissement des tissus au niveau du point d'infection qu'après un mois d'infection environ, au moment où les femelles fécondées sortent des racines. Or, ces symptômes sont caractéristiques de la synthèse de composés phénoliques et, en général, correspondent précisément au développement de la réaction tissulaire (KOSUGE, 1969).

Il ne semble donc pas que la stimulation de l'intensité respiratoire corresponde au déclenchement d'un mécanisme de type hypersensibilité. Par contre, elle survient au moment où de nouvelles racines se développent à partir d'un point d'infection. Elle est importante dans le cas de "Capitole » où de nombreuses racines commencent à se développer ; elle est plus faible, comme l'est également le nombre de racines, dans le cas de "Loros ». Il est probable que la stimulation de la respiration observée corresponde à la reprise d'une activité méristématique importante. De toutes façons, la réaction respiratoire ne correspondrait pas au déclenchement des mécanismes de défense et ne constituerait donc pas le premier facteur de résistance.

En dehors des mécanismes de défense cellulaire tels que l'hypersensibilité, il existe, dans le cas des attaques de nématodes, un facteur particulier pouvant jouer un grand rôle dans l'expression de la résistance. Il est en effet établi que, une fois la larve installée dans les tissus, on assiste au développement autour du parasite d'un syncytium, gaine de cellules géantes et 
multinucléees, qui paraît nécessaire à la nutrition du nématode ainsi qu'au développement plus particulier des femelles (KAPLAN \& KEEN, 1980 ; GOMMERS, 1981 ; GIEBEL, 1982). Il a d'ailleurs été établi que, dans le cas de "Loros ", la présence du syncytium était retardée et moins importante (LAVAIL, comm. pers.). Or les résultats rapportés ici montrent, en début de développement, une capacité de croissance 2 fois plus faible pour "Loros " que pour " Capitole ». De plus, au $8^{\mathrm{e}} \mathrm{j}$, quand la réaction des tissus est à son maximum, le métabolisme des racines est encore ralenti (45 p. 100). Ce ralentissement important dans l'activité métabolique pourrait expliquer la formation retardée du syncytium ou tout au moins y contribuer. Ce processus pourrait intervenir de manière importante dans l'expression de la résistance des tissus.
Les résultats de ce travail suggèrent donc que l'infection des tissus des racines du cv. "Capitole » n'entraîne pas d'effets inhibiteurs irréversibles, mais conduit seulement à un retard dans le développement du système racinaire. Dans ce cas, les conséquences néfastes de l'infection seraient plutôt dues pour cette lignée au retard cumulatif de croissance, conséquence d'infections multiples. Par contre, pour le cv. " Loros ", il semble, tout au moins au stade plantule, que le ralentissement important du métabolisme, ajouté à une capacité de croissance plus faible, puisse jouer un rôle important dans l'expression de la résistance.

\section{RÉFÉRENCES BIBLIOGRAPHIQUES}

Beckman C. H., 1964. Host response to vascular infection. Annu. Rev. Phytopathol., 2, 231-252.

Caubel G., Person F., Rivoal R., 1980. Les nématodes dans les rotations céréalières. Perspect. Agric., 36, 34-47.

Daly J. M., 1976. The carbon balance of diseased plants : changes in respiration, photosynthesis and translocation, p. 450-474. In Heitfuss R. \& Williams P. H., Physiological plant pathology, Springer-Verlag, Berlin, Heidelberg, New York, 890 p.

Davies K. A., Fisher J. M., 1976. Factors influencing the number of larvae of Heterodera avenae invading barley seedlings in vitro. Nematologica, 22, 153-162.

Davy de Virville J., Lance C., Doussinault G., 1982. Réponse respiratoire au cours des premiers stades de l'infection chez diverses lignées de Triticinées sensibles ou résistantes à Pseudocercosporella herpotrichoides (Fron) Deighton. Cryptog. Mycol., 4, 319-332.

Giebel J., 1982. Biochemical interactions between nematodes and plants and their relevance to control. Annu. Rev. Phytopathol., 20, 257-279.

Goodman R. N., Kiraly Z., Zaitlin M., 1967. The biochemistry and physiology of infectious plant disease, p. 66-101, D. Van Nostrand Company Inc., Princeton, 354 p.

Gommers F. J., 1981. Biochemical interactions between nematodes and their relevance to control. Helmintholog. Abstr., 50, 9-21.

Jahier J., 1978. Etude des relations hôte-parasite, aux stades plantule et adulte, chez les Triticinées, dans le cas de Cercosporella herpotrichoides Fron, agent du piétin-verse, Thèse Doct. Ing., Ec. Ntle Supér. Agron. Rennes, 87 p.

Kaplan D. T., Keen N. T., 1980. Mechanisms conferring plant incompatibility to nematodes. Rev. Nématol., 3, 123-134.

Kosuge T., 1969. The role of phenolics in host response to infection. Annu. Rev. Phytopathol., 7, 195-222.
Person F., Doussinault G., 1978. Influence de la température et des caractères des races d'Heterodera avenae Woll. sur la validité d'un test en conditions contrôlées utilisable en sélection des céréales. Ann. Amélior. Plant., 28, 513-527.

Person-Dedryver F., Pannetier D., 1982. Les cultivars d'orge, facteurs de discrimination des pathotypes d'Heterodera avenae. Bull. OEPP, 12, 393-398.

Ritter M., 1972. Place et importance des nématodes dans l'agriculture. Phytoma, 235, 12-22.

Ritter M., 1982. Importance des nématodes à kystes des céréales. Bull. OEPP, 12, 307-316.

Rivoal R., 1977. Identification des races biologiques du nématode à kystes des céréales, Heterodera avenae Woll. en France. Ann. Zool. Ecol. Anim., 9, 261-272.

Rivoal R., 1978. Biologie d'Heterodera avenae Woll. en France. I. Différences dans les cycles d'éclosion et de développement des 2 races FR1 et FR4. Rev. Nématol., 1, 171-179.

Rivoal R., 1979. Biologie d'Heterodera avenae en France. II. Etude des différences dans les conditions thermiques d'éclosion des races FR1 et FR4. Rev. Nématol., 2, 233-248.

Rivoal R., Person F., Caubel G., Scotto la Massèse C., 1978. Méthodes d'évaluation de la résistance des céréales au développement des nématodes : Ditylenchus dipsaci, Heterodera avenae, Pratilenchus spp. Ann. Amelior. Plant., 28, 371-394.

Sosa Moss C., 1966. Contribution à l'étude d'un nématode phytoparasite. Thèse d'Université, Univ. Pierre-et-Marie-Curie, Paris, $149 \mathrm{p}$.

Wheeler H., 1975. Plant Pathogenesis, p. 56-62, Springer-Verlag, Berlin, Heidelberg, New York, 106 p. 